\title{
ARTICLE Distal mutation V486M disrupts the catalytic activity of DPP4 by affecting the flap of the propeller domain
}

\author{
Teng-teng $\mathrm{Li}^{1,2}$, Cheng Peng ${ }^{3,4}$, Ji-qiu Wang ${ }^{5}$, Zhi-jian $\mathrm{Xu}^{3,4}$, Ming-bo Su${ }^{1}$, Jia $\mathrm{Li}^{1,2}$, Wei-liang Zhu ${ }^{3,4}$ and Jing-ya $\mathrm{Li}^{1}$
}

Dipeptidyl peptidase-4 (DPP4) plays a crucial role in regulating the bioactivity of glucagon-like peptide-1 (GLP-1) that enhances insulin secretion and pancreatic $\beta$-cell proliferation, making it a therapeutic target for type 2 diabetes. Although the crystal structure of DPP4 has been determined, its structure-function mechanism is largely unknown. Here, we examined the biochemical properties of sporadic human DPP4 mutations distal from its catalytic site, among which V486M ablates DPP4 dimerization and causes loss of enzymatic activity. Unbiased molecular dynamics simulations revealed that the distal V486M mutation induces a local conformational collapse in a $\beta$-propeller loop (residues 234-260, defined as the flap) and disrupts the dimerization of DPP4. The "open/closed" conformational transitions of the flap whereby capping the active site, are involved in the enzymatic activity of DPP4. Further site-directed mutagenesis guided by theoretical predictions verified the importance of the conformational dynamics of the flap for the enzymatic activity of DPP4. Therefore, the current studies that combined theoretical modeling and experimental identification, provide important insights into the biological function of DPP4 and allow for the evaluation of directed DPP4 genetic mutations before initiating clinical applications and drug development.

Keywords: DPP4; distal mutation; enzymatic activity; molecular dynamics simulation; structure-function mechanism

Acta Pharmacologica Sinica (2022) 43:2147-2155; https://doi.org/10.1038/s41401-021-00818-x

\section{INTRODUCTION}

Dipeptidyl-peptidase 4 (DPP4) is a multifunctional type II cell surface glycoprotein that is widely expressed on epithelial cells, fibroblasts, and leukocyte subsets [1]. The appearance of DPP4 in circulation reveals its dual existence as both membranebound and soluble isoforms, both of which have enzymatic activity [1, 2]. The cleavage of membrane-bound DPP4 could produce a soluble form (amino acids 39-766). As a member of the serine peptidase subfamily $\mathrm{S} 9 \mathrm{~B}$, which includes fibroblastactivation protein a (FAP), resident cytoplasmic proteins (DPP8 and DPP9), and nonenzymatic members (DPP6 and DPP10) $[1,3,4]$, DPP4 cleaves and inactivates several incretin hormones, chemokines, and cytokines. Therefore, it is involved in various physiological processes, including endocrine systems, cancer, inflammation, and immune function $[1,5]$. The functional cleavage of dipeptides by DPP4 occurs at the $\mathrm{N}$-terminus of substrates by specifically cleaving off the $\mathrm{X}$-proline and $X$-alanine dipeptides from the substrates [6]. To date, the bestcharacterized substrates of DPP4 are glucagon-like peptide-1 (GLP-1) and gastric inhibitory polypeptide (GIP), which are responsible for the incretin effect or $60 \%$ of insulin secreted in response to nutrients. Li et al. showed that DPP4 could rapidly inactive GLP-1 (half-life of $2 \mathrm{~min}$ ) and GIP (half-life of 2-3 min) [7]. Therefore, inhibiting the enzymatic activity of DPP4 will prolong the half-life of GLP-1, which provides a strategy to treat type 2 diabetes mellitus (T2DM) [8].

The crystal structure of human DPP4 has revealed that DPP4 adopts a homodimeric architecture, of which each monomeric subunit is comprised of an $\alpha / \beta$ hydrolysis domain and a $\beta$-propeller domain [9]. The $\alpha / \beta$ hydrolysis domain consists of residues $39-51$ and 506-766, including the catalytic triad (S630, D708 and H740). The $\beta$-propeller domain (residues 55-497) consists of eight blades, and each blade is made up of four antiparallel $\beta$-strands [10]. A propeller loop (residues 234-260) from propeller blade 4 is involved in both dimerization and tetramerization interactions [11]. Three residues (Y238, Y248, and Y256) in the propeller loop that locate the interface of dimer have been identified their importance in the dimerization of DPP4 [12]. However, the mechanism of action of the propeller loop in the dimerization and enzymatic activity of DPP4 remains largely unclear. Considering the complicated function of DPP4, it is imperative to delineate the manner in which its enzymatic activity could be regulated. However, using experimental methods, such as X-ray crystallography, to observe the structural dynamics of DPP4 is technically challenging and expensive.

Recent studies [13-18] have revealed a growing interest in the theoretical prediction of protein structure-function relationships by using molecular dynamics (MD) simulations together with

\footnotetext{
${ }^{1}$ State Key Laboratory of Drug Research, the National Drug Screening Center, Shanghai Institute of Materia Medica, Chinese Academy of Sciences, Shanghai 201203, China; ${ }^{2}$ School of Life Science and Technology, ShanghaiTech University, Shanghai 201210, China; ${ }^{3}$ CAS Key Laboratory of Receptor Research; Drug Discovery and Design Center, Shanghai Institute of Materia Medica, Chinese Academy of Sciences, Shanghai 201203, China; ${ }^{4}$ School of Pharmacy, University of Chinese Academy of Sciences, Beijing 100049, China and ${ }^{5}$ Department of Endocrinology and Metabolism, China National Research Center for Metabolic Diseases, National Key Laboratory for Medical Genomes, Ruijin Hospital, Shanghai Jiao Tong University School of Medicine (SJTUSM), Shanghai 200025, China

Correspondence: Wei-liang Zhu (wlzhu@simm.ac.cn) or Jing-ya Li (jyli@simm.ac.cn)

These authors contributed equally: Teng-teng Li, Cheng Peng
}

Received: 15 August 2021 Accepted: 6 November 2021

Published online: 14 December 2021 
experimental validation. In theory, MD simulations can investigate protein dynamics at the atomic level and achieve very fine temporal resolution. Importantly, such simulations can capture how proteins respond to perturbations such as ligand binding/ unbinding, protonation, or mutations. For example, accumulating evidence indicates that MD simulations can identify key protein ligands [19-21], which is valuable in lead optimization. Many studies have also successfully determined the impact of mutations on protein mechanical properties by using MD simulations $[17,18]$. Recently, Maxwell et al. revealed over 50 cryptic epitopes by $0.1 \mathrm{~s}$ simulations of the SARS-CoV-2 proteome [22]. To date, MD simulations of DPP4 have been used for virtual screening and exploring the binding mechanism of inhibitors [23, 24]. As summarized in several reviews [13-16], major improvements in MD sampling efficiency have expanded the impact of biomolecular simulations in drug discovery and design, which has increased the appeal of theoretical simulations for researches.

Previously, a novel DPP4 mutation, p.V486M (c.1456G>A) and other seven sporadic DPP4 variants (D65E, P290L, A291P, S323L, T351A, R492K, and K554Q) were identified in our in-home database [25]. All eight mutations are far from the catalytic site, but even so, the V486M proband showed an extremely high GLP-1 level and reduced enzymatic activity of DPP4 [25]. To determine the functional mechanisms of these distal mutations, in this study, biochemical characterization and MD simulations were combined and applied to the wild-type and eight DPP4 variants. The results revealed that the "open/closed" transition of a flap in the $\beta$ propeller domain (residues 234-260) plays a crucial role in the enzymatic activity of DPP4. The V486M mutant induces a local conformational collapse of the flap and disrupts the dimerization of DPP4. Furthermore, site-directed mutagenesis of the residues located in the flap or interacting with the flap verified the importance of flap movement for the enzymatic activity of DPP4. Therefore, this study uncovered important aspects associated with DPP4 enzymatic activity and DPP4 gene variations in humans, which also offers a powerful combined approach of theoretical prediction and experimental validation for understanding the structure-function mechanism of biomolecules.

\section{MATERIALS AND METHODS}

Single-site-directed mutagenesis

The DNA encoding the DPP4 extracellular domain (residues 39-766) was amplified by PCR from the full-length DPP4 gene and subsequently cloned into a modified vector pFastBac1 with six His residues at the C-terminus. Twelve DPP4 mutations, including eight mutations identified from individuals (D65E, P290L, A291P, S323L, T351A, V486M, R492K, and K554Q) of which the detailed information refers to our previous study [25] and four mutations for confirmatory research (K122D, D243A, P257A, and S664P) were introduced into DPP4 by using phanata master mix DNA polymerase (Vazyme Biotech, catalog number: P515, Nanjing, China), followed by digestion of the template DNA with Dpn I (Thermo Fisher Scientific, catalog number: ER1702, Waltham, MA, USA).

\section{Protein expression and purification}

The extracellular domain of human DPP4 (residues 39-766) was expressed using a Bac-to-Bac baculovirus expression system. In brief, the DNA encoding hDPP4 was cloned into the EcoR I and Hind III sites of pFastBac1. The final construct contains the $\mathrm{N}$-terminal melittin signal peptide to facilitate secretion and a C-terminal $6 \times$ histidine tag for purification. The constructed DNA was then transformed into bacterial DH10Bac competent cells (Weidi Biotechnology, catalog number: DE1070, Shanghai, China), and the recombined bacmid DNA was extracted and transfected into Sf9 cells using Cellfectin II Reagent (Thermo
Fisher Scientific, catalog number: 10362100, Waltham, MA, USA). Recombinant baculovirus was generated by transposition using the Bac-to-Bac system. The virus was used to infect High Five cells. The secreted glycosylated recombinant protein was isolated from the cell culture supernatant containing the target protein and harvested $72 \mathrm{~h}$ after infection. The target protein was loaded onto nickel (Ni)-charged resin (GE Healthcare, catalog number: 17-5247, Little Chalfont, Bukinghamshire, UK) and eluted with $0.25 \mathrm{M}$ imidazole.

\section{Nonreducing and reducing SDS-PAGE}

The recombinant DPP4 was mixed with nonreducing SDS sample buffer $(50 \mathrm{mM}$ Tris- $\mathrm{HCl}, \mathrm{pH} 6.8,2 \%$ SDS, $10 \%$ glycerol, $0.1 \%$ bromophenol blue) or reducing SDS sample buffer (50 mM Tris$\mathrm{HCl}, \mathrm{pH} 6.8,2 \%$ SDS, $10 \%$ glycerol, $0.1 \%$ bromophenol blue, $1.5 \%$ dithiothreitol) and incubated at $100^{\circ} \mathrm{C}$ for $10 \mathrm{~min}$. The samples and protein marker were loaded onto the gels. Following electrophoresis, the gels were stained overnight using Coomassie blue. Bands were quantitated using Image Lab 6.0.

\section{Enzyme assays}

DPP4 activity was assayed by a continuous fluorometric assay, which is cleaved by the enzyme to release fluorescent aminomethylcoumarin (AMC). Liberation of AMC was monitored using an excitation wavelength of $355 \mathrm{~nm}$ and an emission wavelength of $460 \mathrm{~nm}$ using an Envision microplate reader (PerkinElmer, Waltham, Massachusetts, USA). DPP4 activity was assayed in $100 \mathrm{mM}$ HEPES buffer ( $\mathrm{pH}$ 7.5) containing $0.1 \mathrm{mg} / \mathrm{mL}$ BSA (buffer A) in a total volume of $50 \mu \mathrm{L}$. All kinetic constants were computed by direct fits of the data to the appropriate nonlinear equation using GraphPad Prism 8.0 software. Substrates, Gly-Pro-AMC, were obtained from GL Biochem (Shanghai, China). Omarigliptin was obtained from Selleck Chemicals (Houston, Texas, USA).

\section{Thermal shift}

Purified DPP4, wild type (WT) or variants, was diluted to $0.25 \mathrm{mg} /$ $\mathrm{mL}(0.7 \mu \mathrm{M})$ in buffer A containing $5 \times$ SYPRO orange dye (Thermo Fisher Scientific, catalog number: S5692, Waltham, MA, USA) and $25 \mathrm{mM}$ inhibitor (dissolved in DMSO). The samples were heated from $25^{\circ} \mathrm{C}$ to $95^{\circ} \mathrm{C}$ at a rate of $1{ }^{\circ} \mathrm{C} / \mathrm{min}$, and the fluorescence signals were monitored by a real-time PCR system (Agilent Technologies, Santa Clara, CA, USA).

\section{Molecular dynamic simulation}

Starting with the X-ray structure (PDB ID: 2QT9) [26] by deleting its inhibitor, we evaluated and compared the conformational dynamics of the wild type and DPP4 mutants. The missing residues were built by SWISS-MODEL [27], and the protein was mutated by PyMOL software [28]. The protonation states were predicted by the $\mathrm{H}++$ web server using $\mathrm{pH} 7.5$ [29]. The Amber 99SB*-ILDNP [30] force field was used to parameterize the protein. Each system was solvated into a cubic box of TIP3P water extended by $10 \AA$. A rational number of counter ions of $\mathrm{Na}^{+}$or $\mathrm{Cl}^{-}$ were added to neutralize the system. Finally, the total number of atoms in each system was $\sim 80,000$. All bonds involving hydrogen atoms were constrained with the LINCS algorithm [31]. All longrange electrostatic interactions were calculated with the particle mesh Ewald [32] method with a $12 \AA$ cut-off.

Each system was first minimized using the steepest descent algorithm to remove bad contacts, followed by $0.5 \mathrm{~ns}$ heating from $0 \mathrm{~K}$ to $300 \mathrm{~K}$ and $2 \mathrm{~ns}$ equilibrium simulations with constraints $\left(10 \mathrm{kcal} \cdot \mathrm{mol}^{-1} \cdot \AA^{-2}\right)$ on heavy atoms. Finally, a $1 \mu \mathrm{s}$ production simulation on each system at $300 \mathrm{~K}$ was performed with GROMACS5.1.4 software. To identify the conformational change of the flap, distance analysis was carried out by the g_distance routine (GROMACS). The hydrogen bond interactions of each system were analyzed along the $1 \mu$ s MD simulations. 

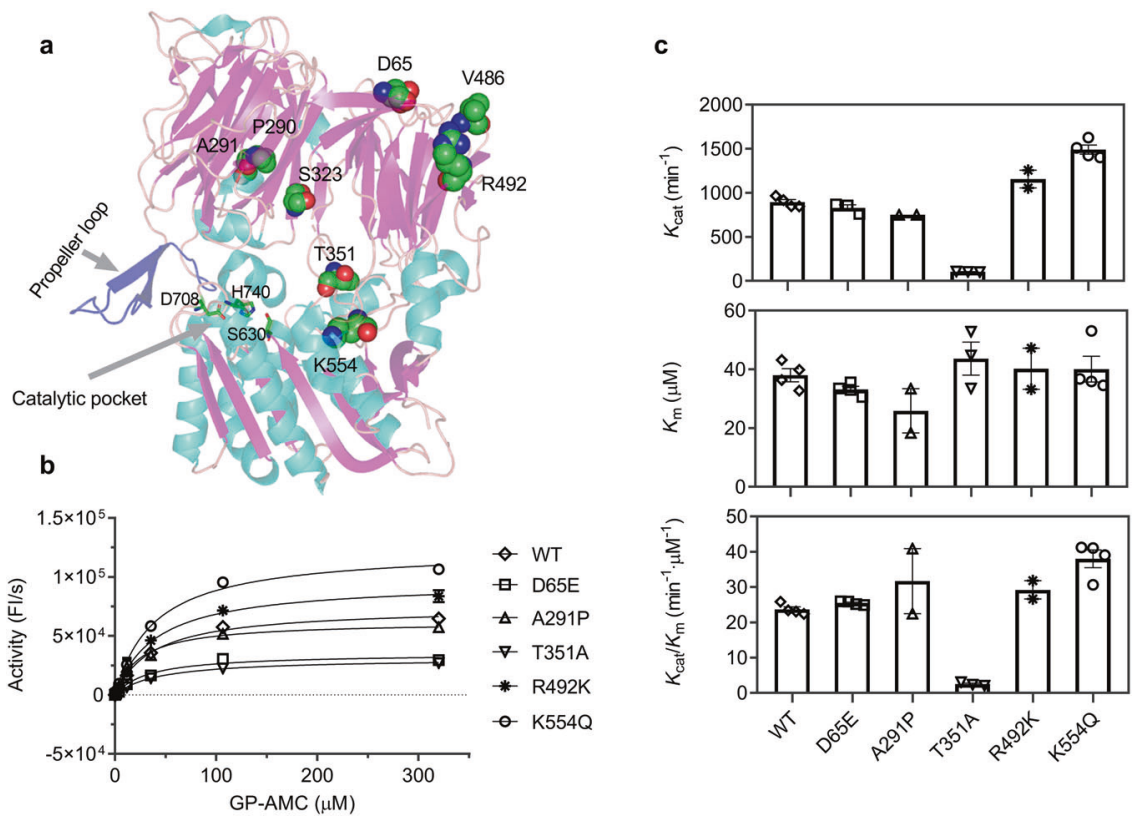

Fig. 1 Overview of DPP4 mutant sites and kinetic curves of mutants. a The structure of DPP4 (PDB ID: 2QT9). Mutant sites were shown in spheres. The catalytic triad (S630, D708 and H740) was shown in sticks. The propeller loop (P234-G260, defined as flap in below text) was colored in marine. b, c Dipeptidyl peptidase activity of WT and mutants was determined with GlyPro-AMC as a substrate in the enzymatic activity assay. $K_{\mathrm{m}}$ curve of DPP4 mutation (b) and means and standard deviations of kinetic constants are plotted (c).

\begin{tabular}{|llll|}
\hline Table 1. & Enzymatic constants of DPP4-WT and mutants. \\
\hline Mutants & $K_{\mathrm{m}}(\mu \mathrm{M})$ & $K_{\text {cat }}\left(\mathrm{min}^{-1}\right)$ & $K_{\text {cat }} / K_{\mathrm{m}}\left(\mathrm{min}^{-1} \cdot \mu \mathrm{M}^{-1}\right)$ \\
\hline WT & $38.02 \pm 4.48$ & $894.87 \pm 58.15$ & $23.54 \pm 1.50$ \\
D65E & $33.18 \pm 2.04$ & $845.41 \pm 58.44$ & $25.48 \pm 0.55$ \\
P290L & $\mathrm{ND}^{\mathrm{a}}$ & $\mathrm{ND}$ & $\mathrm{ND}$ \\
A291P & $30.07 \pm 4.72$ & $769.33 \pm 26.92$ & $25.58 \pm 13.01$ \\
S323L & $\mathrm{ND}$ & $\mathrm{ND}$ & $\mathrm{ND}$ \\
T351A & $43.67 \pm 9.73$ & $104.40 \pm 3.10$ & $2.39 \pm 0.51$ \\
V486M & $\mathrm{ND}$ & $\mathrm{ND}$ & $\mathrm{ND}$ \\
R492K & $40.22 \pm 9.90$ & $1156.30 \pm 141.32$ & $28.75 \pm 3.67$ \\
K554Q & $40.07 \pm 8.72$ & $1490.58 \pm 101.75$ & $37.20 \pm 5.01$ \\
\hline
\end{tabular}

Mean \pm SD values are shown.

${ }^{a} \mathrm{ND}$ indicates not detected.

\section{RESULTS}

A genetically distal mutation, V486M, ablates the catalytic activity of the DPP4 enzyme

In our previous study, a novel DPP4 mutation (p.V486M) was identified in a proband who had low DPP4 enzymatic activity and high GLP-1 levels, together with two other family members who were carriers [25]. Meanwhile, additional seven sporadic DPP4 mutations, viz., D65E, P290L, A291P, S323L, T351A, R492K, and $\mathrm{K} 554 \mathrm{Q}$, were further verified from our in-home population database [25]. All eight sporadic DPP4 mutations were far from the catalytic pocket, with the shortest distance of $23.7 \AA$ for K554 (Fig. 1a). To comprehensively evaluate the effects of the genetic DPP4 distal mutations on its enzymatic activities, we expressed all eight His-tagged DPP4 distal mutants in High Five cells, and they were subjected to biochemical assays after expression and purification.

By determining the enzyme kinetic constants $\left(K_{\mathrm{m}}, K_{\mathrm{cat}}\right.$ and $K_{\text {cat }} / K_{\mathrm{m}}$ ) of the DPP4 mutants, we found that the V486M mutant showed complete loss of catalytic activity (Table 1). In addition, P290L and S323L also exhibited blunted activity. A significant drop by approximately 10-fold in the second-order rate constant $K_{\text {cat }} /$ $K_{\mathrm{m}}$ was observed in the T351A mutant, whereas a small increase $\left(\sim 1.7\right.$-fold) in $K_{\text {cat }}$ was observed in the K554Q mutant (Fig. $1 \mathrm{~b}$ and Table 1).

V486M retains thermal stability and substrate binding ability but disrupts DPP4 dimerization

To determine whether the distal mutation introduced in this study affects the overall conformational stability, we measured the thermal stability of the DPP4 mutants by monitoring their melting temperature $\left(T_{m}\right)$. Surprisingly, the V486M mutant showed $T_{m}$ values equivalent to those of wild-type DPP4 (Fig. 2a), indicating that the protein backbone of the V486M mutant was not disturbed.

Moreover, the $\mathrm{N}$-terminus of the substrates is essential since they have to be recognized and bonded. The DPP4 crystal structure has revealed how substrate specificity is achieved, after which it preferentially cleaves after Xaa-Pro or Xaa-Ala (Xaa being any amino acid) [10]. To confirm the substrate binding of the V486M mutant, we applied a thermal shift assay by using omarigliptin to mimic the substrate binding mode. The DPP4 inhibitor omagliptin binds rapidly and competitively to the active site of DPP4, which is a reversible and highly selective process [33]. In wild-type DPP4, the $T_{\mathrm{m}}$ values increased by 12.41 degrees compared to the DMSO control. Similarly, the $T_{\mathrm{m}}$ values increased by 13.79 degrees in the V486M mutant. In addition, we found that there was no significant change in substrate binding of the V486M mutant and wild-type DPP4 (Fig. 2b, c). Taken together, the ablated enzymatic activity of the V486M mutant was clearly not a result of active site collapse or the entire protein.

DPP4 has a monomeric glycoprotein subunit that homodimerizes to acquire enzymatic activity. The formation of dimers is the prerequisite for DPP4 to exert enzymatic activity [9]. Measured by nonreduced SDS-PAGE, we explored the possibility that loss of activity of the V486M mutant was associated with dimer formation. Interestingly, the V486M mutant resulted in an $\sim 18.1 \%$ reduction in the dimer relative to the wild type (Fig. $2 \mathrm{~d}, \mathrm{e}$ ). However, which factor contributes to the dimer formulation of V486M is unknown. 


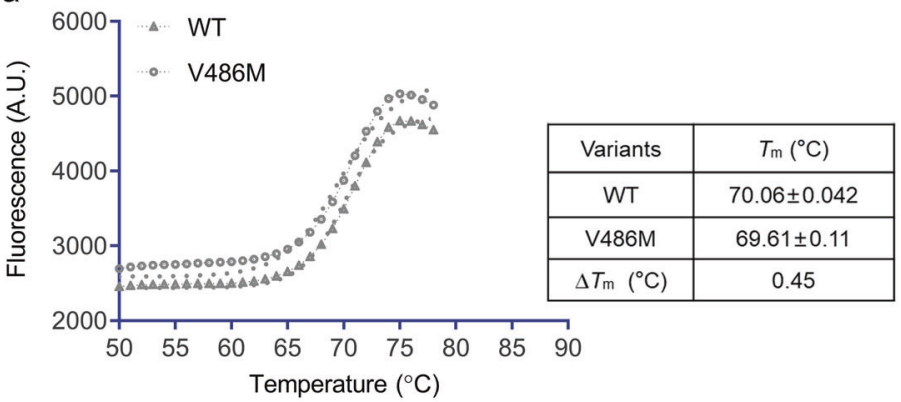

b

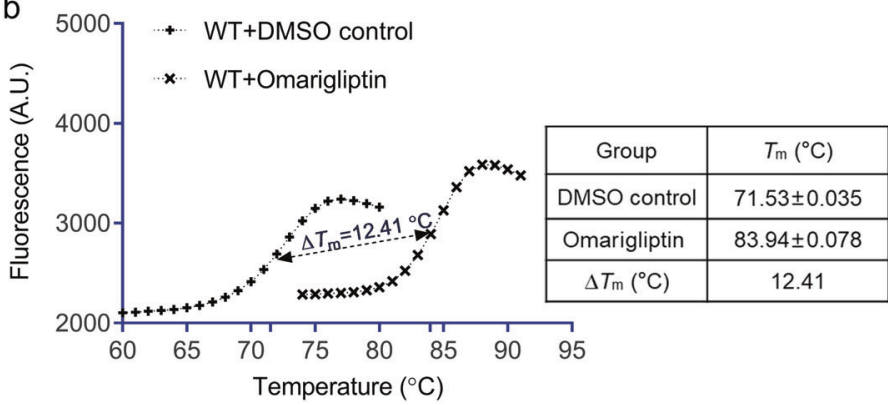

$\mathrm{C}$

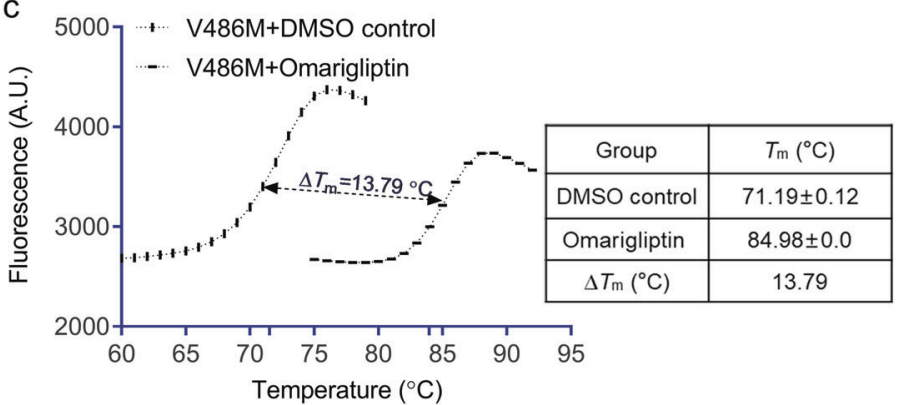

d
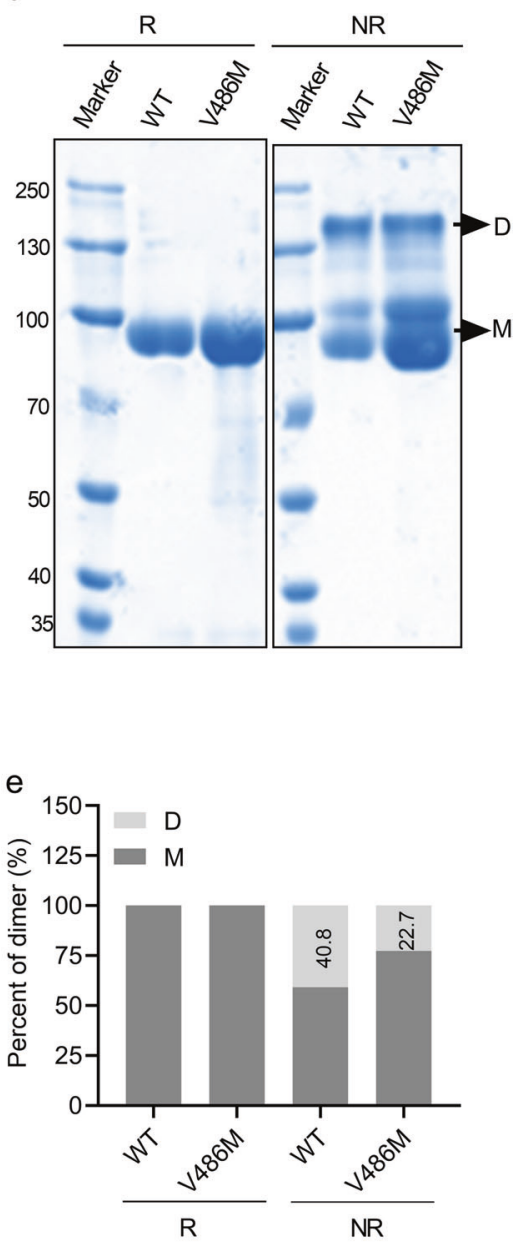

Fig. 2 Biochemical properties of DPP4-WT and DPP4-V486M. a Stability comparison of WT and V486M. $\Delta T_{\mathrm{m}}$ is indicated the difference between mean value of WT and V486M. Mean \pm SD values are shown. Inhibitors binding activity comparison of WT (b) and V486M (c) in the absence or presence of DPP4 inhibitor omargliptin. $\Delta T_{\mathrm{m}}$ is indicated the difference between mean value of DMSO control and Omarigliptin group. Mean \pm SD values are shown. d, e Coomassie blue-stained reducing SDS-PAGE and nonreducing SDS-PAGE (10\%) of WT and V486M. e The dimer percent statistics of WT and V486M. The percent $40.8 \%$ and $22.7 \%$, indicates dimer percent of WT and V486M, respectively. R, NR, $D, M$, presents reducing SDS-PAGE, nonreducing SDS-PAGE, dimer, and monomer.

Molecular dynamics simulation reveals that the propeller loop, namely, the flap, mediates DPP4 enzymatic activity

V486 lies in strand 3 blade $8(\beta 8 / 3)$ of the $\beta$-propeller domain of DPP4, which is distant from the dimerization interface and catalytic triad ( $45.2 \AA)$. Therefore, it is unlikely that V486 directly impacts the dimerization or enzymatic activity of DPP4. However, the detailed mechanism by which $\mathrm{V} 486 \mathrm{M}$ remotely regulates enzymatic activity is unclear. To understand this mechanism, we performed long-time unbiased MD simulations to study the conformational dynamics of V486M at the atomic level compared with wild-type DPP4. As shown in Fig. 3, we found that the propeller loop (residues 234-260) is flexible in wild-type DPP4, switching between two distinct conformations controlled by the propeller loop, "closed" and "open"; therefore, we defined it as a flap. During $1 \mu \mathrm{s}$ MD simulations, the wild-type DPP4 underwent two-time transitions between "closed" and "open", at approximately $540 \mathrm{~ns}$ and $900 \mathrm{~ns}$, respectively. However, in the V486M mutant, the flap was relatively rigid and remained "open" during the MD simulations, indicating that the conformational transition of the flap is important for the enzymatic activity of DPP4.

To further evaluate the relationship between this conformational transition and enzymatic activity, we analyzed the conformational transition of the flap in other seven DPP4 mutants. As shown in Fig. 4, for the two mutants that lost enzymatic activity (P290L and S323L), their flaps also remained in an "open" state. For the other mutants that maintain DPP4 enzymatic activity, their flaps are flexible between "closed" and "open"; for example, R492K underwent three transitions between "closed" and "open", at approximately $20 \mathrm{~ns}, 400 \mathrm{~ns}$ and $450 \mathrm{~ns}$, respectively. Therefore, the results showed that the conformational switch between the "closed" and "open" states of the flap is necessary for the enzymatic activity of DPP4.

Mutagenesis of S664P results in monomeric DPP4 without catalytic activity

To validate the importance of the flap's conformational transition on enzymatic activity, we analyzed and compared the hydrogen bond interactions formed by the flap in its "closed" and "open" structures. As shown in Fig. 5a, the hydrogen bond formed by K122 and D243 could stabilize the "closed" conformations, whereas the hydrogen bond formed by P257 and S664, which lies in the root of the flap, could be found in both the "closed" and "open" conformations. In particular, the hydrogen bond between P257 and S664 was formed by their main chain atoms. Therefore, 
a

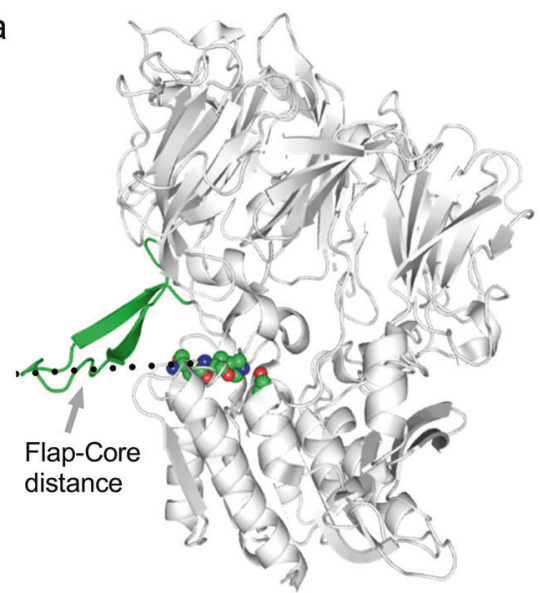

b

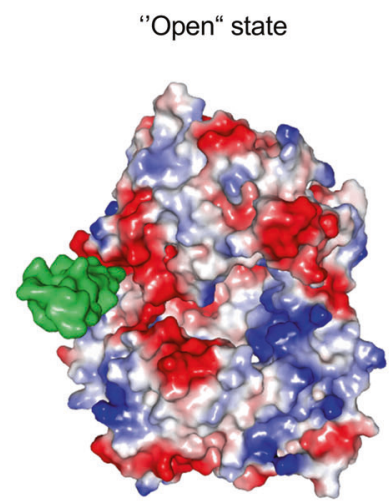

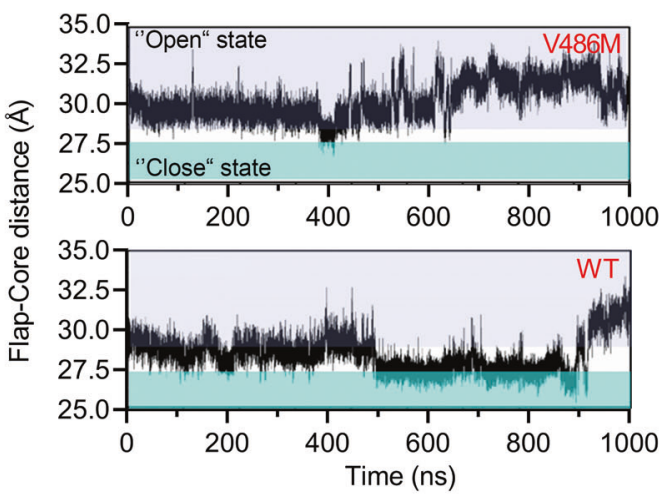

"Close" state

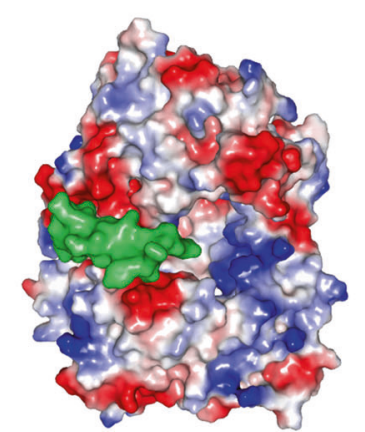

Fig. 3 Analysis of the conformational changes of DPP4-WT and DPP4-V486M. a The mass center distance between the tip of flap (D243-L246) and catalytic triad (Flap-Core distance) was calculated to evaluate the conformational changes of the flap. The conformations with Flap-Core distance less than $27.5 \AA$ refer to "close" state, and the conformations with Flap-Core distance greater than $28.4 \AA$ refer to "open" state. b Representative structures of the "close" and "open" states.

we designed a mutagenesis study of these two hydrogen bonds. For D243-K122 interactions, the D243A mutant destabilizes the "closed" conformations, thus maintaining enzymatic activity as a control, whereas the K122D mutant prevents DPP4 from shifting from "open" to "closed", thus decreasing enzymatic activity. For the P257-S664P interactions, the P257A mutant retains the interactions to maintain enzymatic activity as a control, whereas the S664P mutant disturbs the interactions to decrease enzymatic activity. Therefore, we designed four mutants (K122D, D243A, P257A, and S664P) that are in the flap or interact with this region.

We next performed enzyme kinetics studies to validate the efficacy of the four mutants. The results showed that among the four mutations, the S664P single-site mutation abolished the enzymatic activity of DPP4, and K122D significantly decreased $K_{\text {cat }} / K_{\mathrm{m}}$ by approximately $50 \%$, indicating that conformational transitions of the flaps were indeed critical for the enzymatic activity of DPP4. In addition, D243A and P257A retained some of the enzymatic activity of DPP4 (Fig. 5b, c and Table 2).

The flap lies in the dimer interface, and disrupting the "open/ closed" conformational transitions may affect the dimer formation of DPP4. To confirm whether such transitions affect the dimer formation, we employed a nonreduced SDS-PAGE assay. The single mutation, S664P, disrupted DPP4 dimerization with a concomitant loss of enzymatic activity, while other three mutations had no change compared with the wild type (Fig. 5d). These results indicated that the "open/closed" conformational transitions of the flap, to some extent, were associated with dimer formation. During $1 \mu \mathrm{s}$ MD simulations, we found that the flap of S664P is kept in the "open" conformation and we also sampled many unstable conformations resulting from the disturbance of the interactions between S664P and V665/N263 (Fig. 6a, b). Therefore, these results indicated that $\mathbf{S 6 6 4}$ possibly supports the overall protein backbone and that the S664P mutation may disturb the overall protein structure and stability. To confirm this hypothesis, the structural stability of the S664P mutant was further assayed by thermal shift. Consistent with the prediction of the MD simulation, the $T_{\mathrm{m}}$ values of the S664P mutant dramatically dropped by 25 degrees (Fig. 6c).

In summary, we found that the movement is critical for the dimerization and consequently the enzymatic activity of DPP4, as illustrated by mutagenesis tests of S664P and K122D. Furthermore, the collapse of the conformational transition of the flap in the human DPP4 mutation V486M, together with P290L and S323L, led to a loss of enzyme activity.

\section{DISCUSSION}

DPP4 is recognized as an important T2DM therapeutic target [34]. It is a protease with a ubiquitous expression profile and it is involved in many physiological processes, including glucose homeostasis [35], inflammation [36-38], and tumor growth $[39,40]$. Circulating levels of DPP4 activity and sDPP4 are increased in humans with T2DM [41], suggesting that inhibiting the activity of DPP4 could be used as a treatment for T2DM. There are a few studies about DPP4 genetic alterations and their association with T2DM and other diseases. Bouchard et al. reported DPP4 polymorphisms associated with blood pressure, lipids, and diabetes-related phenotypes in obese patients [42]. In 

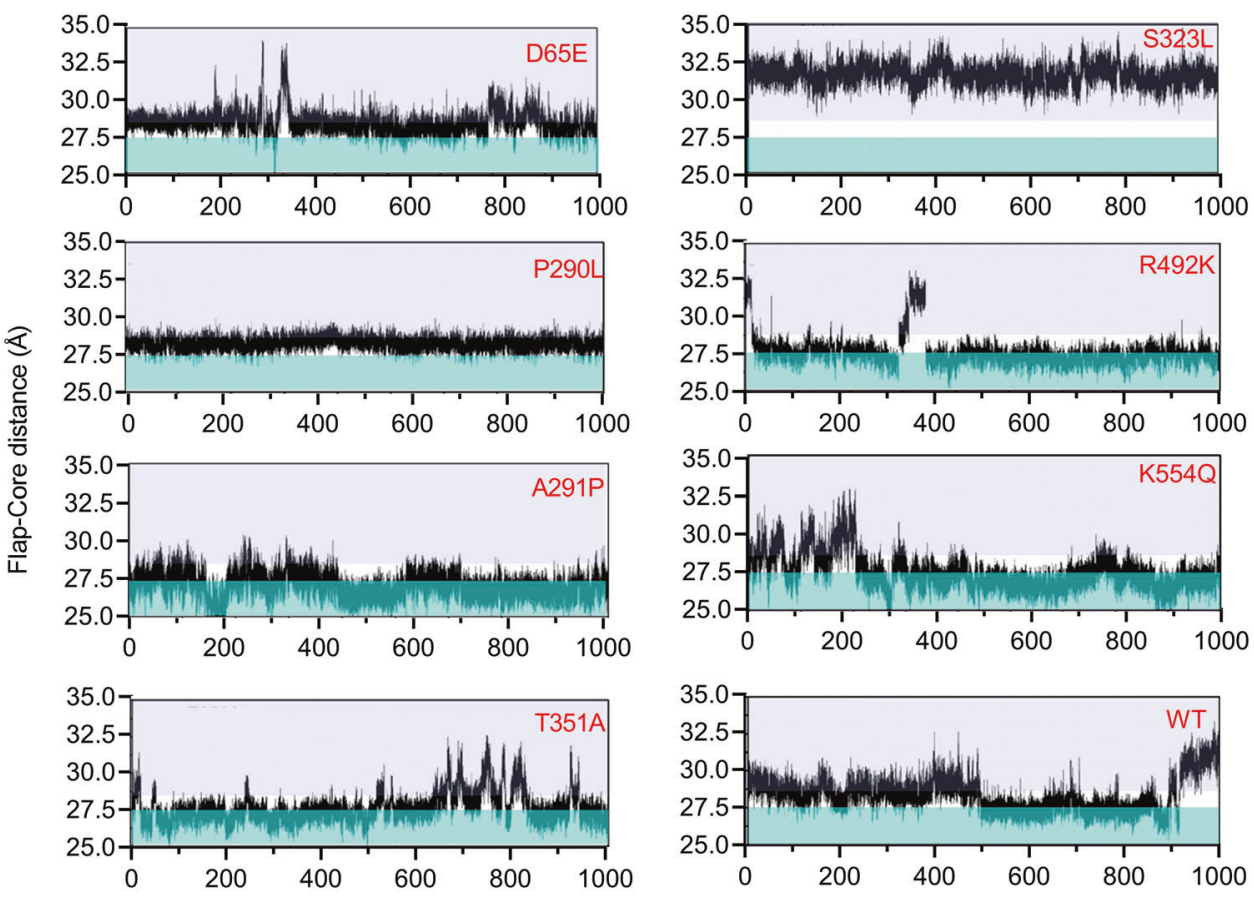

Time (ns)

Fig. 4 Analysis of the conformational changes of DPP4-WT and other seven mutants. The mass center distance between the tip of flap (D243-L246) and catalytic triad (Flap-Core distance) was calculated to evaluate the conformational changes of the flap. The conformations with Flap-Core distance less than $27.5 \AA$ refer to "close" state, and the conformations with Flap-Core distance greater than $28.4 \AA$ refer to "open" state.

addition, single nucleotide polymorphisms rs6741949 in the DPP4 gene interacts with body adiposity and negatively affects GLP-1 levels, insulin secretion, and glucose tolerance [43]. It is unclear that biofunction of the entire lost activity of DPP4 in humans, although ablation of DPP4 activity has been studied in both rat and mouse models. C57BL/6 DPP4-KO mice exhibited enhanced glucose tolerance, lower plasma glucose, and higher plasma insulin [44]. The long-term physiological effects of loss of function of DPP4 have not yet been reported.

In our work, we followed up on the continuous changes in plasma glucose, lipid metabolism and tumor markers in the proband who carried the V486M mutation. Surprisingly, the proband did not report any hypoglycemic events despite high GLP-1 levels over the past 6 years (data not shown). This discrepancy might be due to certain physiological compensations to maintain glucose homeostasis at the whole organism level. For the V486M carrier proband, a half dosage reduction of DPP4 activity seems to be relatively benign, at least through 46 years old. This result suggests that long-term inhibition of DPP4 activity may have no serious adverse effects. Thus, the functional variations in DPP4 may have important clinical significance. In addition, this study can help an understanding of the biochemical properties of D65E, P290L, A291P, S323L, T351A, V486M, R492K, and $\mathrm{K} 554 \mathrm{Q}$ mutations in Chinese individuals.

The enzymatic activity of DPP4 was found to be influenced by mutations that lie far from the active site. The finding of the "open/closed" conformational transitions of the flap domain neighboring the active site explained the functional mechanism of the distal mutations in our MD simulations. The flap domain is highly conserved among the S9B subfamily. Previously, Rasmussen et al. crystallized the DPP4 structure and revealed that this domain stabilizes the dimeric structure and may act as a lid on the side entrance to the catalytic pocket [10]. In particular, residues Y238, Y248, and Y256 in the flap domain are essential to DPP4 dimerization [12]. Similar to DPP4, its close gene family member
Prolyl oligopeptidase (POP), has loop A (residues 189-209) and loop B (residues 577-608) at the domain interface implicated in substrate entry to the active site [45]. However, exactly how this domain impacts DPP4 dimerization and enzymatic activity remains unknown.

To understand the structure-function mechanism at the atomic level, we applied MD simulations together with experimental validation. Observation of the conformational dynamics of DPP4 mutants in MD simulations revealed that the flap (residues 234-260) regulates enzymatic activity. We showed that the "open/ closed" conformational transitions of the flap are of significance for the enzymatic activity of DPP4. Furthermore, according to our hydrogen bond analyses, four DPP4 mutants were designed to validate the necessity of the flap conformational transitions for enzymatic activity, viz. K122D, D243A, P257A, and S664P. The K122D and S664P mutants were designed to decrease DPP4 enzymatic activity, while the D243A and P257A mutants were designed as controls that retained enzymatic activity. Enzyme kinetics experiments validated the predictions of the four mutants, especially the S664P mutant, which abolished the enzymatic activity of DPP4, revealing the reliability of our MD simulations. Further simulations of S664P revealed that the flap remained in the "open" state, again suggesting the necessity of the conformational transition of the flap. In summary, molecules that reduce or even disrupt the conformational transition have the potential to be developed for the treatment of T2DM.

Subsequently, residue S664 was found to be essential for the enzymatic activity and dimerization of DPP4. The nonreduced SDS-PAGE assay revealed a disrupted dimerization of S664P, and the thermal shift assay showed decreased $T_{\mathrm{m}}$ values, indicating that S664 might play a crucial role in DPP4 protein stability. During the MD simulations, S664P maintains a stable conformation except for the flap region. As a result, conformational changes in the flap distort the substrate binding pocket, which then leads to significantly reduced enzymatic activity directly or indirectly. 
a

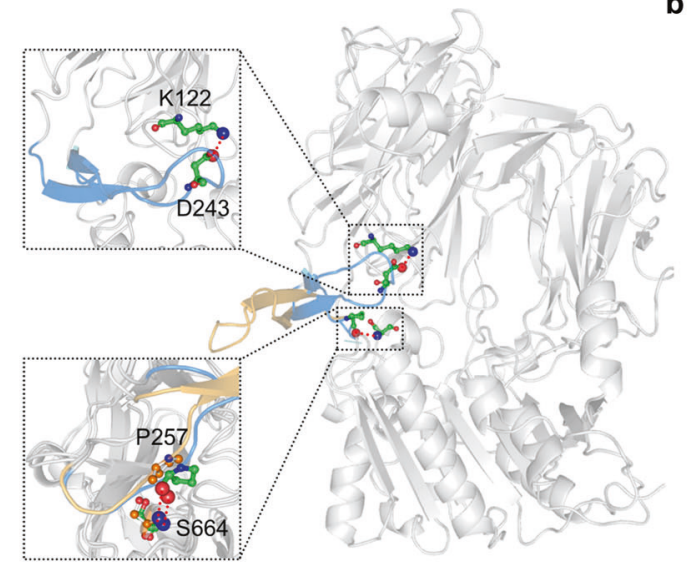

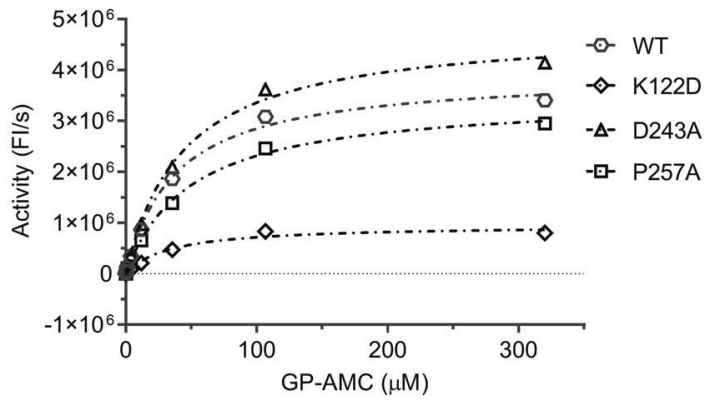

C

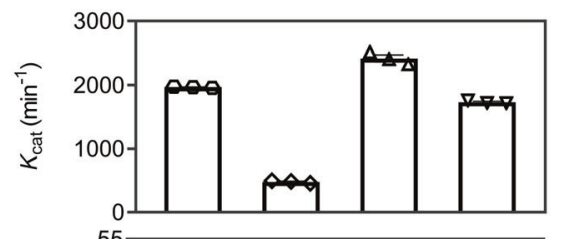

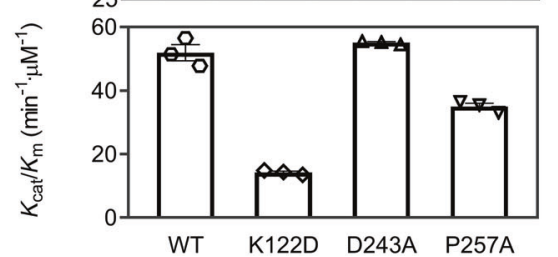

d

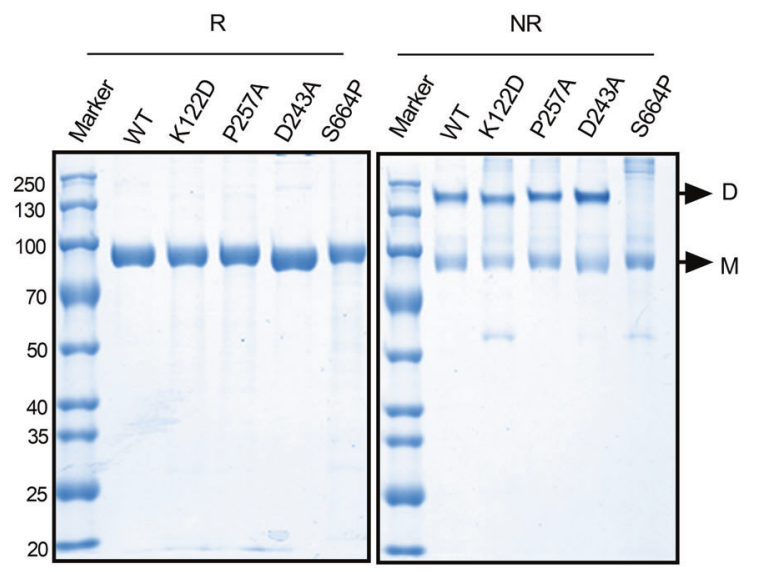

Fig. 5 Biochemical properties of flap mutation. a Structural analysis of "close" states (blue domain) and "open" states (orange domain). The hydrogen bonds were labeled in red dotted line. Enzymatic activity analysis of Flap mutations. $K_{m}$ curve of flap mutation (b) and means and standard deviations of kinetic constants are plotted (c). d Analysis of dimerization of flap mutation by nonreducing SDS-PAGE. Coomassie blue-stained reduced SDS-PAGE and nonreduced SDS-PAGE (10\%) of flap mutations. R, NR, D, M indicates reducing SDS-PAGE, nonreducing SDS-PAGE, dimer, and monomer.

\begin{tabular}{|llcl|}
\hline Table 2. & \multicolumn{3}{l|}{ Enzyme kinetic constants of flap variants. } \\
\hline Variants & $K_{\mathrm{m}}(\mu \mathrm{M})$ & \multicolumn{1}{c|}{$K_{\mathrm{cat}}\left(\mathrm{min}^{-1}\right)$} & $K_{\mathrm{cat}} / K_{\mathrm{m}}\left(\mathrm{min}^{-1} \cdot \mu \mathrm{M}^{-1}\right)$ \\
\hline WT & $37.96 \pm 2.03$ & $1965.38 \pm 31.70$ & $47.77 \pm 4.38$ \\
K122D & $33.60 \pm 3.86$ & $477.59 \pm 16.15$ & $13.46 \pm 0.70$ \\
P257A & $49.55 \pm 1.80$ & $1730.80 \pm 20.27$ & $36.46 \pm 1.75$ \\
D243A & $43.87 \pm 2.31$ & $2415.70 \pm 39.79$ & $55.57 \pm 0.55$ \\
S664P & ND $^{\mathrm{a}}$ & $\mathrm{ND}$ & $\mathrm{ND}$ \\
\hline
\end{tabular}

Mean \pm SD values are shown.

anD indicates not detected.

Indeed, kinetic assays suggested that S664P has undetectable enzymatic activity. Additionally, the V486M mutant also had an "open" flap during the MD simulations, which may be further explained by the findings in the flap crystal structure of the V486M protein.

According to Fig. 4, the conformational transition frequency of the flap might be associated with the enzymatic activity of DPP4. For example, in the wild type and R492K mutant, the flap underwent two and three transitions between "closed" and "open" states, respectively. Enzyme kinetics experiments revealed that R492K has similar catalytic activity to the wild type. However, in the $\mathrm{K} 554 \mathrm{Q}$ mutant, the flap underwent over ten conformational transitions. Correspondingly, the catalytic activity of the $\mathrm{K} 554 \mathrm{Q}$ mutant was higher than that of the wild type. These results suggest that there might be a positive correlation between conformational transition frequency and catalytic activity. Therefore, our current findings provide new insights into developing novel inhibitors that block dimerization and activators that either open or remove this flap. Our method of a combination of MD simulation and experimental validation for describing the structure-function mechanism of DPP4 may be helpful for studying other key enzymes.

\section{CONCLUSION}

DPP4 is recognized as a crucial therapeutic target for T2DM and more than twenty inhibitors were already approved for type 2 diabetes therapy. With the Human Genome Project progresses to the whole genome sequence of the population, varieties of single nucleotide polymorphism were identified. Eight sporadic DPP4 variants (D65E, P290L, A291P, S323L, T351A, V486M, $\mathrm{R} 492 \mathrm{~K}$, and $\mathrm{K} 554 \mathrm{Q}$ ) that are distant from both the catalytic 

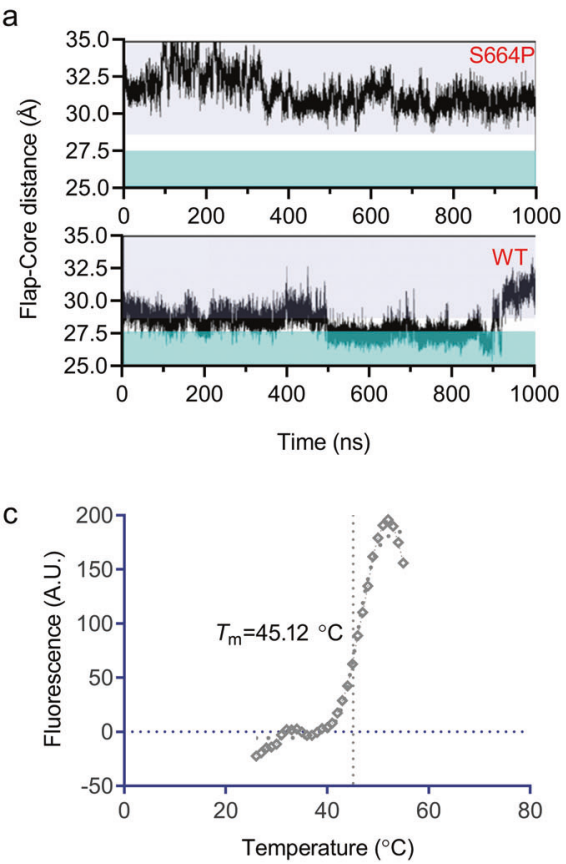

b
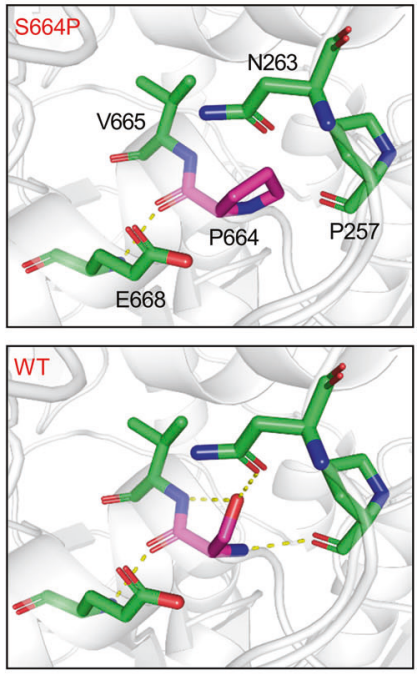

Fig. 6 Analysis of the conformational changes of DPP4-WT and DPP4-S664P. a The mass center distance between the tip of flap (D243L246) and catalytic triad (Flap-Core distance) was calculated to evaluate the conformational changes of the flap. $\mathbf{b}$ Representative structures of the DPP4-WT and DPP4-S664P, the hydrogen bonds were shown as yellow dotted line. c Thermal stability of S664P mutant in DMSO. The $T_{\mathrm{m}}$ of S664P mutant was $45.12^{\circ} \mathrm{C}$, and $T_{\mathrm{m}}$ of wild type control was $70.06^{\circ} \mathrm{C}$ in the front data.

pocket and the dimerization interface, were identified in Chinese population. In addition, the V486M mutant showed a reduced enzymatic activity. To determine the functional mechanisms of these distal mutations, in this study, biochemical characterization and MD simulations were combined and applied to the wild-type and eight DPP4 variants. The results showed that the single point mutations that damage activity of DPP4 could cause conformational transition failure of the propeller flap and disrupts the dimerization of DPP4. Further site-directed mutagenesis of K122D, D243A, P257A, and S664P predicted by $M D$ simulations verified the importance of the flap's conformational dynamics for DPP4 enzymatic activity. In addition, the conformational transition frequency of the flap might be associated with the enzymatic activity of DPP4. Therefore, this finding may have implications for understanding the structure-function mechanism of DPP4 and open an avenue for the development of specific DPP4 inhibitors that may have diagnostic and therapeutic applications in humans.

\section{ACKNOWLEDGEMENTS}

This work was supported by the National Natural Science Foundation of China (No. 92057116), the National Science and Technology Major Project (2018ZX09711002018), the Strategic Priority Research Program of Chinese Academy of Sciences grant (XDA12040204), the Shanghai Commission of Science and Technology (18431900900), and National Key R\&D Program of China (2016YFA0502301 and 2017YFB0202601). The molecular dynamics simulations were partially run at the TianHe 1 supercomputer in Tianjin and TianHe 2 supercomputer in Guangzhou.

\section{AUTHOR CONTRIBUTIONS}

JYL and WLZ put forward to conception and contributed to research design. TTL planned and carried out the biochemistry experiments and analyzed the data. MBS participated in the enzymatic experiments. CP performed molecular dynamics simulations and analyzed the data. ZJX participated in the molecular dynamics simulations experimental design. JQW contributed clinical flowing-up data of DPP4V486M carrier and project discussion. JL contributed reagents and analytic tools. TTL and $C P$ contributed to writing the paper, and JYL and WLZ reviewed/edited the paper.

\section{ADDITIONAL INFORMATION}

Competing interests: The authors declare no competing interests.

\section{REFERENCES}

1. Rohrborn D, Wronkowitz N, Eckel J. DPP4 in diabetes. Front Immunol. 2015;6:386.

2. Zhong J, Maiseyeu A, Davis SN, Rajagopalan S. DPP4 in cardiometabolic disease: recent insights from the laboratory and clinical trials of DPP4 inhibition. Circ Res. 2015;116:1491-504.

3. Gorrell MD. Dipeptidyl peptidase IV and related enzymes in cell biology and liver disorders. Clin Sci. 2005;108:277-92.

4. Deacon CF. Physiology and pharmacology of DPP-4 in glucose homeostasis and the treatment of type 2 diabetes. Front Endocrinol. 2019;10:80.

5. Gorrell MD, Gysbers V, McCaughan GW. CD26: a multifunctional integral membrane and secreted protein of activated lymphocytes. Scand J Immunol. 2001;54:249-64.

6. Elmansi AM, Awad ME, Eisa NH, Kondrikov D, Hussein KA, Aguilar-Perez A, et al. What doesn't kill you makes you stranger: dipeptidyl peptidase-4 (CD26) proteolysis differentially modulates the activity of many peptide hormones and cytokines generating novel cryptic bioactive ligands. Pharmacol Ther. 2019;198:90-108.

7. Li N, Wang LJ, Jiang B, Li XQ, Guo CL, Guo SJ, et al. Recent progress of the development of dipeptidyl peptidase-4 inhibitors for the treatment of type 2 diabetes mellitus. Eur J Med Chem. 2018;151:145-57.

8. Trzaskalski NA, Fadzeyeva E, Mulvihill EE. Dipeptidyl peptidase-4 at the interface between inflammation and metabolism. Clin Med Insights Endocrinol Diabetes. 2020;13:1179551420912972.

9. Chien $\mathrm{CH}$, Huang LH, Chou CY, Chen YS, Han YS, Chang GG, et al. One site mutation disrupts dimer formation in human DPP-IV proteins. J Biol Chem. 2004;279:52338-45.

10. Rasmussen HB, Branner S, Wiberg FC, Wagtmann N. Crystal structure of human dipeptidyl peptidase IV/CD26 in complex with a substrate analog. Nat Struct Biol. 2003;10:19-25.

11. Engel M, Hoffmann T, Wagner L, Wermann $M$, Heiser $U$, Kiefersauer $R$, et al. The crystal structure of dipeptidyl peptidase IV (CD26) reveals its functional regulation and enzymatic mechanism. Proc Natl Acad Sci USA. 2003;100:5063-68.

12. Tang HK, Chen KC, Liou GG, Cheng SC, Chien CH, Tang HY, et al. Role of a propeller loop in the quaternary structure and enzymatic activity of prolyl dipeptidases DPP-IV and DPP9. FEBS Lett. 2011;585:3409-14.

13. Do $P C$, Lee $E H$, Le L. Steered molecular dynamics simulation in rational drug design. J Chem Inf Model. 2018;58:1473-82. 
14. Liu X, Shi D, Zhou S, Liu H, Liu H, Yao X. Molecular dynamics simulations and novel drug discovery. Expert Opin Drug Discov. 2018;13:23-37.

15. Wade RC, Salo-Ahen $\mathrm{OMH}$. Molecular modeling in drug design. Molecules. 2019;24:321.

16. Hollingsworth SA, Dror RO. Molecular dynamics simulation for all. Neuron. 2018;99:1129-43.

17. McCoy MD, Shivakumar V, Nimmagadda S, Jafri MS, Madhavan S. SNP2SIM: a modular workflow for standardizing molecular simulation and functional analysis of protein variants. BMC Bioinforma. 2019;20:171.

18. Azadi S, Tafazzoli-Shadpour M, Omidvar R. Steered molecular dynamics simulation study of quantified effects of point mutation induced by breast cancer on mechanical behavior of E-cadherin. Mol Biol. 2018;52:723-31.

19. Zhang C, Feng LJ, Huang Y, Wu D, Li Z, Zhou Q, et al. Discovery of novel phosphodiesterase-2A inhibitors by structure-based virtual screening, structural optimization, and bioassay. J Chem Inf Model. 2017;57:355-64.

20. Hucke O, Coulombe R, Bonneau P, Bertrand-Laperle M, Brochu C, Gillard J, et al. Molecular dynamics simulations and structure-based rational design lead to allosteric HCV NS5B polymerase thumb pocket 2 inhibitor with picomolar cellular replicon potency. J Med Chem. 2014;57:1932-43.

21. Spahn V, Del Vecchio G, Labuz D, Rodriguez-Gaztelumendi A, Massaly N, Temp J, et al. A nontoxic pain killer designed by modeling of pathological receptor conformations. Science. 2017;355:966-9.

22. Zimmerman MI, Porter JR, Ward MD, Singh S, Vithani N, Meller A, et al. SARS-CoV2 simulations go exascale to predict dramatic spike opening and cryptic pockets across the proteome. Nat Chem. 2021;13:651-9.

23. Meduru H, Wang YT, Tsai JJ, Chen YC. Finding a potential dipeptidyl peptidase-4 (DPP-4) inhibitor for type-2 diabetes treatment based on molecular docking, pharmacophore generation, and molecular dynamics simulation. Int J Mol Sci. 2016;17:920.

24. Hou H, Wang Y, Li C, Wang J, Cao Y. Dipeptidyl peptidase-4 is a target protein of epigallocatechin-3-gallate. Biomed Res Int. 2020;2020:5370759.

25. Zhao DD, Zhao SQ, Wang $X$, Su MB, Liu W, Ma QY, et al. Clinical and physiological characterization of elevated plasma glucagon-like peptide-1 levels (hyperglipemia) in a dipeptidyl peptidase iv mutation carrier. Front Endocrinol. 2018;9:62.

26. Kaelin DE, Smenton AL, Eiermann GJ, He $H$, Leiting $B$, Lyons $K A$, et al. 4-arylcyclohexylalanine analogs as potent, selective, and orally active inhibitors of dipeptidyl peptidase IV. Bioorg Med Chem Lett. 2007;17:5806-11.

27. Waterhouse A, Bertoni M, Bienert S, Studer G, Tauriello G, Gumienny R, et al. SWISS-MODEL: homology modelling of protein structures and complexes. Nucleic Acids Res. 2018;46:W296-W303.

28. DeLano WL. The PyMOL user's manual. San Carlos, CA: DeLano Scientific; 2002. p. 452.

29. Anandakrishnan R, Aguilar B, Onufriev AV. $\mathrm{H}++$ 3.0: automating pK prediction and the preparation of biomolecular structures for atomistic molecular modeling and simulations. Nucleic Acids Res. 2012;40:W537-41.
30. Aliev AE, Kulke M, Khaneja HS, Chudasama V, Sheppard TD, Lanigan RM. Motional timescale predictions by molecular dynamics simulations: case study using proline and hydroxyproline sidechain dynamics. Proteins. 2014;82:195-215.

31. Ryckaert JP, Ciccotti G, Berendsen HJ. Numerical integration of the cartesian equations of motion of a system with constraints: molecular dynamics of n-alkanes. J Comput Phys. 1977;23:327-41.

32. Darden T, York D, Pedersen L. Particle mesh Ewald: An N.log (N) method for Ewald sums in large systems. J Chem Phys. 1993;98:10089-92.

33. Burness CB. Omarigliptin: first global approval. Drugs. 2015;75:1947-52.

34. Holst JJ, Deacon CF. Inhibition of the activity of dipeptidyl-peptidase IV as a treatment for type 2 diabetes. Diabetes. 1998;47:1663-70.

35. Mulvihill EE, Varin EM, Gladanac B, Campbell JE, Ussher JR, Baggio LL, et al Cellular sites and mechanisms linking reduction of dipeptidyl peptidase-4 activity to control of incretin hormone action and glucose homeostasis. Cell Metab. 2017;25:152-65.

36. Silva Junior WS, Souza M, Kraemer-Aguiar LG. Dipeptidyl peptidase 4 (DPP4), adipose inflammation, and insulin resistance: is it time to look to the hepatocyte? Hepatobiliary Surg Nutr. 2018;7:499-500.

37. Baggio LL, Varin EM, Koehler JA, Cao X, Lokhnygina Y, Stevens SR, et al. Plasma levels of DPP4 activity and sDPP4 are dissociated from inflammation in mice and humans. Nat Commun. 2020;11:3766.

38. Fen Z, Ni Y, Nagashimada M, Nagata N, Xu L, Mukaida N, et al. DPP-4 inhibition by linagliptin attenuates obesity-related inflammation and insulin resistance by regulating M1/M2 macrophage polarization. Diabetes. 2016;65:2966-79.

39. Barreira da Silva R, Laird ME, Yatim N, Fiette L, Ingersoll MA, Albert ML. Dipeptidylpeptidase 4 inhibition enhances lymphocyte trafficking, improving both naturally occurring tumor immunity and immunotherapy. Nat Immunol. 2015;16:850-8.

40. Hollande C, Boussier J, Ziai J, Nozawa T, Bondet V, Phung W, et al. Inhibition of the dipeptidyl peptidase DPP4 (CD26) reveals IL-33-dependent eosinophilmediated control of tumor growth. Nat Immunol. 2019;20:257-64.

41. Varin EM, Mulvihill EE, Beaudry JL, Pujadas G, Fuchs S, Tanti JF, et al. Circulating levels of soluble dipeptidyl peptidase- 4 are dissociated from inflammation and induced by enzymatic DPP4 inhibition. Cell Metab. 2019;29:320-34. e5

42. Bouchard L, Faucher G, Tchernof A, Deshaies Y, Lebel S, Hould FS, et al. Comprehensive genetic analysis of the dipeptidyl peptidase- 4 gene and cardiovascular disease risk factors in obese individuals. Acta Diabetol. 2009:46:13-21.

43. Bohm A, Wagner R, Machicao F, Holst JJ, Gallwitz B, Stefan N, et al. DPP4 gene variation affects GLP-1 secretion, insulin secretion, and glucose tolerance in humans with high body adiposity. PLoS One. 2017;12:e0181880.

44. Marguet D, Baggio L, Kobayashi T, Bernard AM, Pierres M, Nielsen PF, et al Enhanced insulin secretion and improved glucose tolerance in mice lacking CD26. Proc Natl Acad Sci USA. 2000;97:6874-9.

45. Szeltner Z, Juhasz T, Szamosi I, Rea D, Fulop V, Modos K, et al. The loops facing the active site of prolyl oligopeptidase are crucial components in substrate gating and specificity. Biochim Biophys Acta. 2013;1834:98-111. 